\title{
Engaging Physics Tutoring: A didactical toolbox for teaching assistants (TAs)
}

\section{Vira Bondar' ${ }^{1}$, Jonas Nuber², Manuel Zeyen ${ }^{1}$, Guillaume Schiltz ${ }^{3}$, Günther Dissertori ${ }^{1}$}

${ }^{1}$ ETH Zürich, Institute for Particle Physics and Astrophysics, Switzerland, ${ }^{2}$ Paul Scherrer Institute (PSI), Switzerland, ${ }^{3}$ ETH Zürich, Laboratory of Solid State Physics, Switzerland.

\begin{abstract}
In this paper we present a project dedicated to the development of a didactical toolbox of material for teaching assistants (TAs) supervising exercise classes for non-physics majors at ETH Zurich. With our material we were able to support TAs in preparing high-quality exercise sessions for their class which go beyond direct instruction and activate students intellectually and emotionally. The materials are developed for 13 exercise sessions and are presented in the form of an eBook. The materials were immediately used by several TAs in dedicated focus groups. The positive feedback of students and TAs suggests that our material helped to activate and engage the students, enhancing their learning - even in the challenging setting of online teaching.
\end{abstract}

Keywords: Teaching tools; physics; eBook; student engagement; teacher training. 


\section{Introduction}

As a polytechnic university, ETH Zurich has compulsory introductory physics courses in almost all of its bachelor study programs. The Department of Physics is offering a total of 14 different physics courses which are all tailored to the specific needs of the non-physics major programs. In addition to a physics lecture, which typically adresses several hundred students, small-group exercice classes, comprising 25 to 30 students each, are an integral part of the educational offer. The main goal of these exercice classes is to train students in applying the concepts that have been discussed in the lecture. The exercise classes are run by more than 150 teaching assistants (TAs), most of them being PhD students.

In the past, the Department of Physics made a huge effort in reforming its introductory lectures according to well-established educational principles (Meredith \& Redish, 2013; Crouch \& Mazur, 2001). The exercise classes, however, remained almost out of focus. TAs are invited to attend teaching courses which are offered by the central support unit, but the general principles covered by those courses is of little help when TAs have to plan, organize and teach physics in their classes. How can we improve the learning success in physics exercise classes? Which strategies can help to step out of standard automatism such as pure exercise-solving and direct instruction? How can we "hook and hold" the students? With regards to those questions, we have developed a didactical toolbox including a wide range of tailored teaching material for our TAs.

The idea for the EPT (Engaging Physics Tutoring) project arose from our observation that in practice many TAs struggle to implement techniques which they have learnt in general basic teaching courses. Furthermore, the time it takes to prepare an engaging lesson usually exceeds the preparation time which TAs can invest in addition to further duties like correcting homework and managing their class. Therefore, in this project we developed a didactical toolbox of materials for physics TAs and published it as an eBook (Bondar et al., 2021). In 2020 we have used the toolbox as a pilot in 13 exercise classes and we reported how TAs have been using it for improving their instruction. Due to the Covid19 pandemic, half of the exercise classes were taught online.

\section{Approach and developments}

\subsection{Structure of the developed materials}

The toolbox consists of concept questions (Oliveira, 2013), advanced organizers (Gurlitt, 2012) and case-based calculation examples. Additionally, we included ideas for the "hit" of each lesson - which is based on an engaging phenomenon, that involves the students emotionally and fosters their active thinking in order to help them developing higher-level competences. Occasionally we also included "hands-on" experiments that are especially suitable for online performance and activation of the students. 
Most of the material is based on real-life examples in order to motivate students, encourage them for active interaction and engage them emotionally. Figure 1 illustrates some examples of application ideas used in the materials.

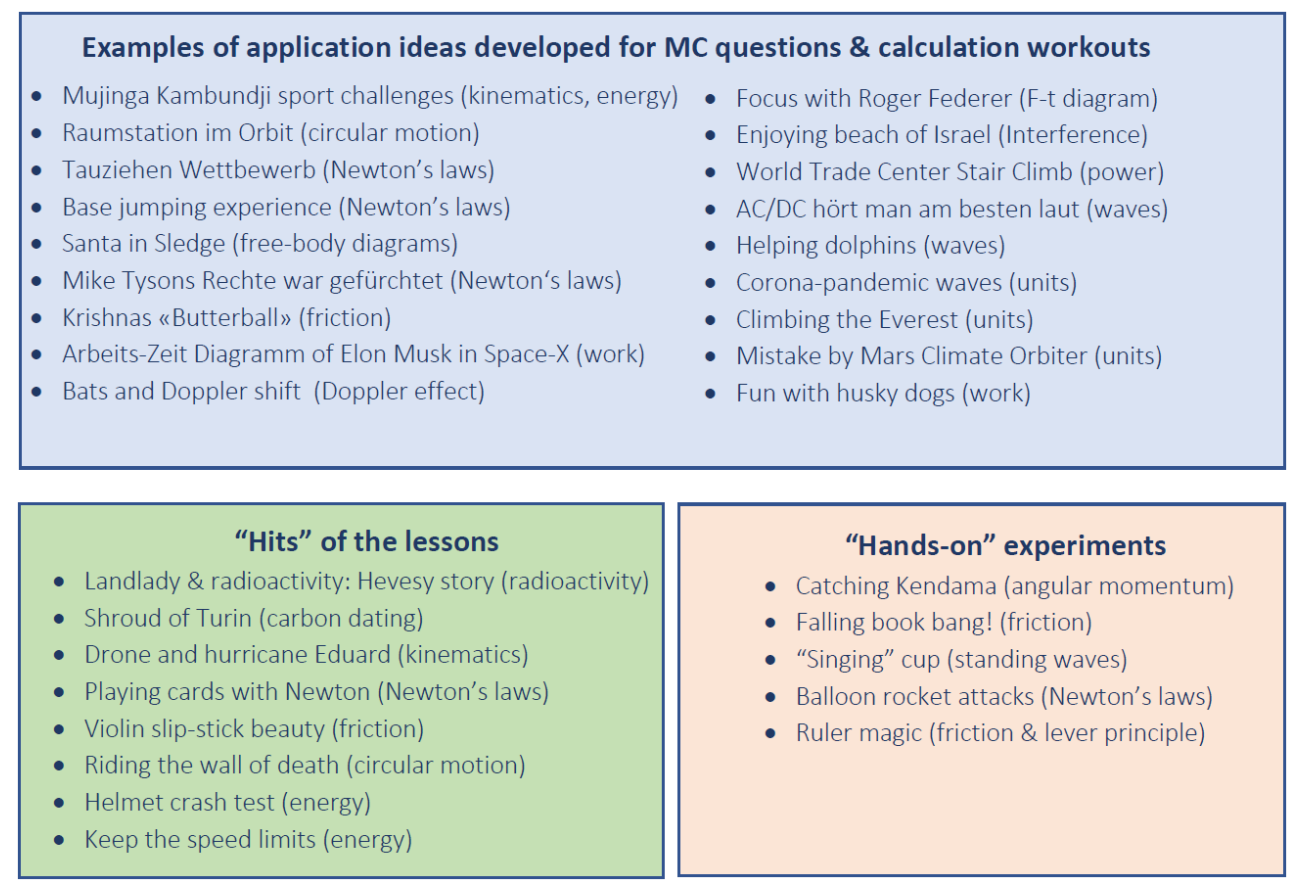

Figure 1. Real-life examples implemented in the materials; $M C=$ Multiple Choice.

\subsection{Didactical approach}

With the toolbox at hand, we encouraged TAs to use our materials relying on a wellestablished approach in the design of their exercise class. Namely, they have to align their planning with specific learning goals of the course, which then define the teaching content and activities of their lessons. Assessment techniques should be applied to inform students and teachers whether a teaching goal has been achieved. This will ensure constructive alignment of all teaching and learning activities (Biggs \& Tang, 2015). 
The developed materials are aligned with learning objectives of the class and can serve as a basis for the design of learning activities and assessment tasks during the exercise session (see Figure 2). In addition to the traditional teaching approach, TAs can develop own activities based on the collaborative and highly interactive SCALE-UP approach (Feldman et al., 2019).

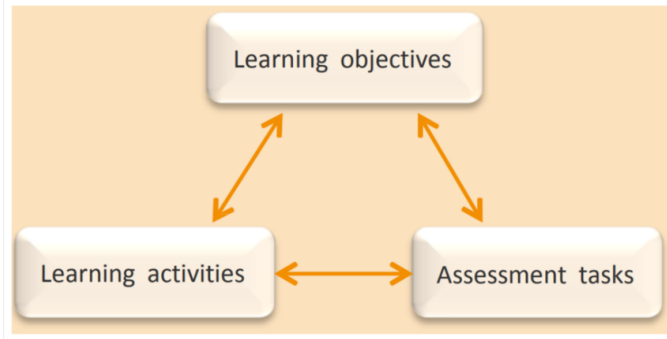

Figure 2. The materials can be used as a basis for the design of Learning activities and assessment tasks.

\subsection{Some Examples}

Here we give some examples of the developed materials; Figure 3 shows examples of reallife applications of the topics of units (Mars orbiter) and of friction (violin vs. squeaky door).
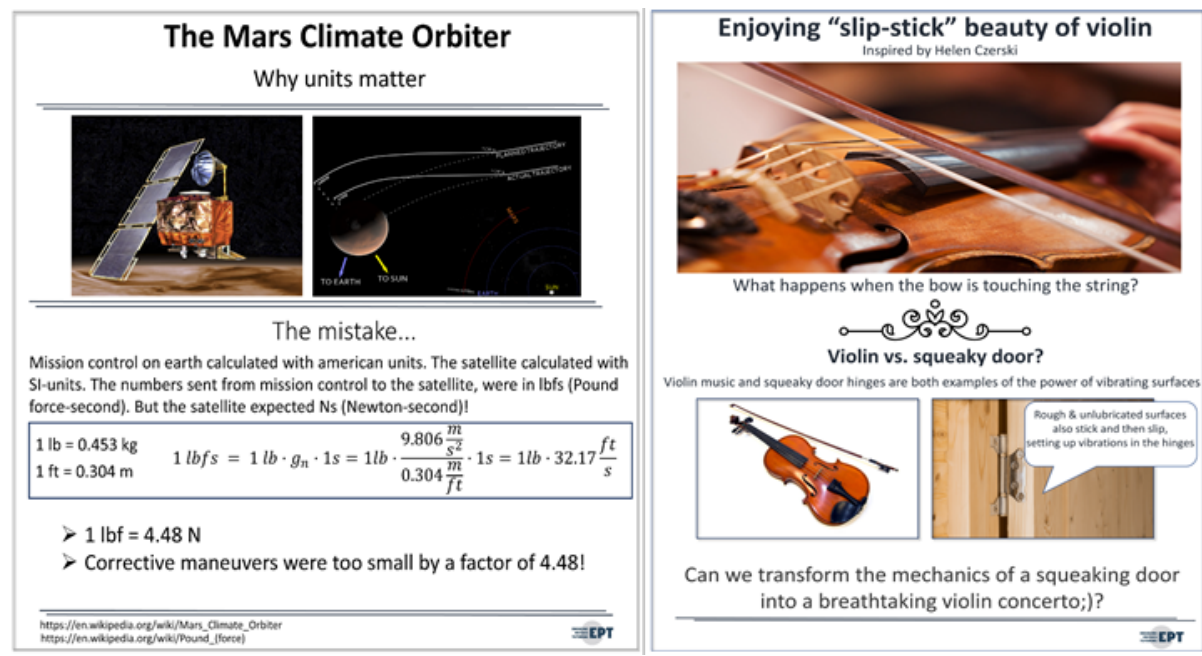

Figure 3. Examples of real-life applications of the topics of units (left) and friction (right).

For students it is also important to have a bird-view of the topics presented during the semester, as it enables them to create a network of knowledge. To help students following links between different topics as well as their common frameworks we also prepared mind maps. These schemes can serve as road-maps while working through the corresponding subtopics. Figure 4 shows an example of a mind map dedicated to kinematics. 


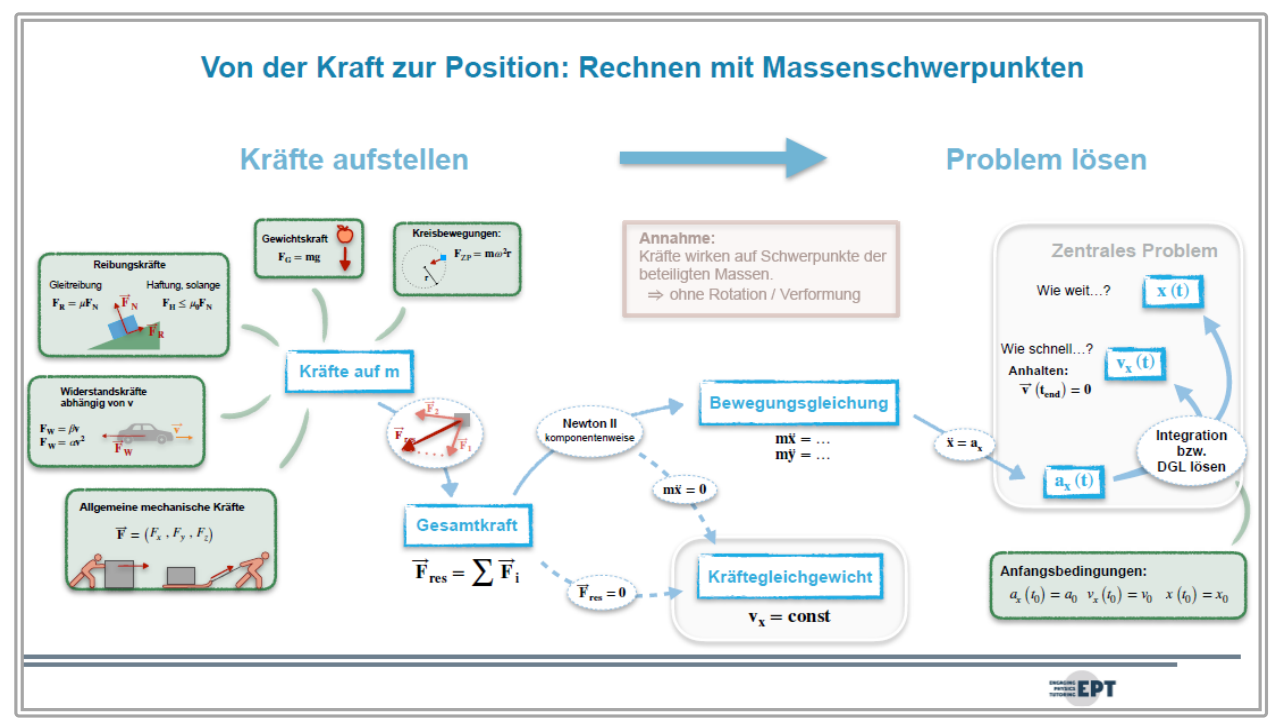

Figure 4. Example of a mind map on kinematics.

\subsection{New developments}

As a new method, we have developed a "2Q" system. At the beginning of each class, the students are asked to answer a set of two Multiple Choice (MC) questions which are closely related to learning objectives of the lesson. The answers are not yet revealed. At the end of the exercise class the students are asked to answer the same questions again, after which the correct answers are discussed. This system allows the TAs not only to assess the learning success in their lesson but also to outline key topics of the lesson and provide a road map for the students.

We got very positive feedback about this system from students (Figures 5, 6, 7, 8) as well as from the TAs (Figures 8 and 9). Furthermore, we observed that the questions activated and engaged the students right from the start of the lesson. Sets of suitable questions for each lesson are included in the toolbox.

\section{Implementation and feedback}

The materials were immediately used by 6 TAs. Namely, we had two dedicated focus groups almost entirely teaching their lessons with our material. Another four TAs were occasionally using the material as ideas / building blocks for the design of their lessons.

In order to estimate the success of the project, we used an online anonymous survey to collect student feedback. The survey was administered to one of our focus groups, where at least 
$90 \%$ of each lesson was based on our materials. Below you will find students' answers to some key questions. A total of 26 students responded to the survey.
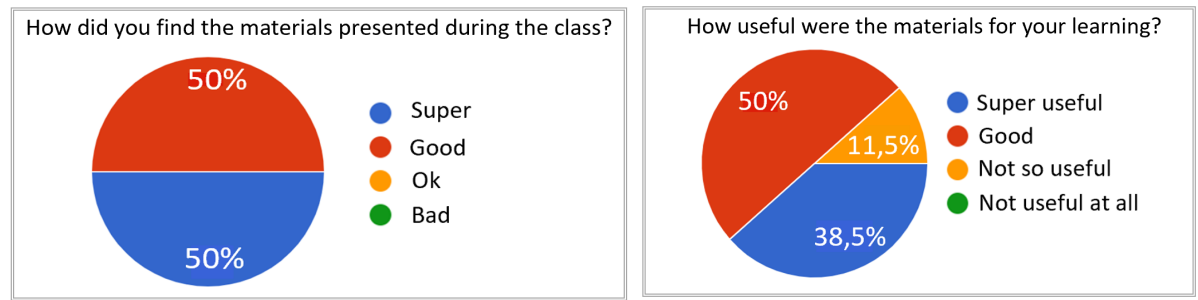

Figure 5. Results of student's feedback on usefulness of EPT materials in their learning $(N=26)$.

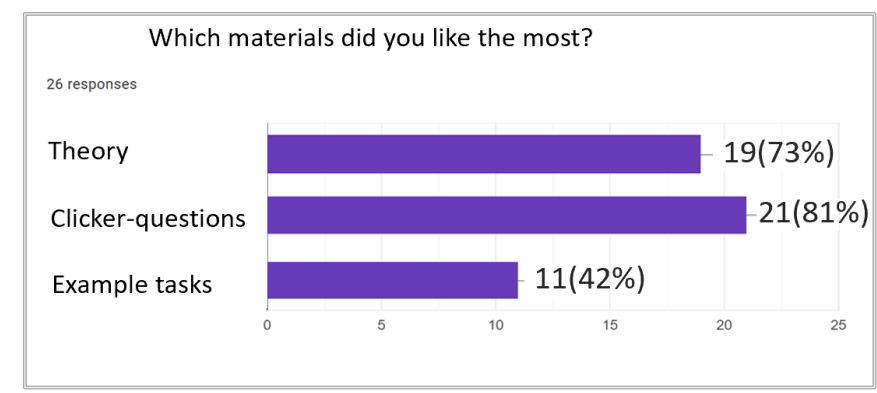

Figure 6. Results of students' feedback on favorite types of EPT material.

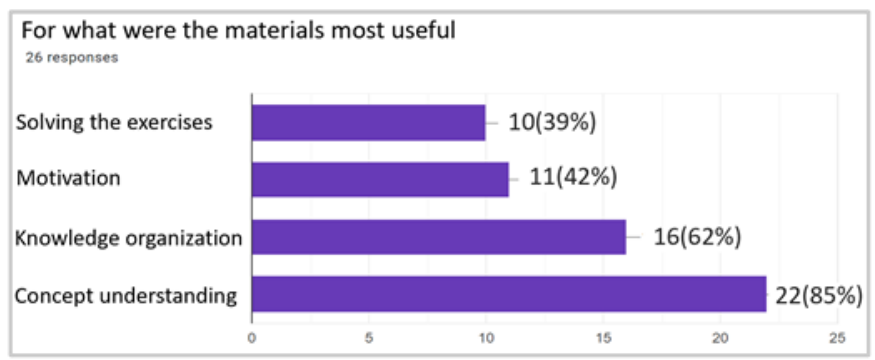

Figure 7. Results of students' feedback on the role of EPT materials in their own learning.

The positive feedback from students and from the TAs suggests that our material helped to activate and engage the students emotionally and to enhance their learning - even in the challenging setting of online teaching.

In particular, the results of the diagrams in Figures 6 and 7 show that on average the students rate the role of the materials for their conceptual understanding higher than for their problemsolving skills. This is particularly interesting since the example exercises were, most of the time, very close to the homework exercises, by using similar ideas and solution paths. The missing competence to transfer knowledge to specific problems is a common issue in the 
learning process of non-experts (Rebello et al., 2007) and is therefore especially relevant for students of introductory courses. The performance of the TAs also plays an important role, which we neglected here. Our evaluated focus group was taught by an experienced TA.

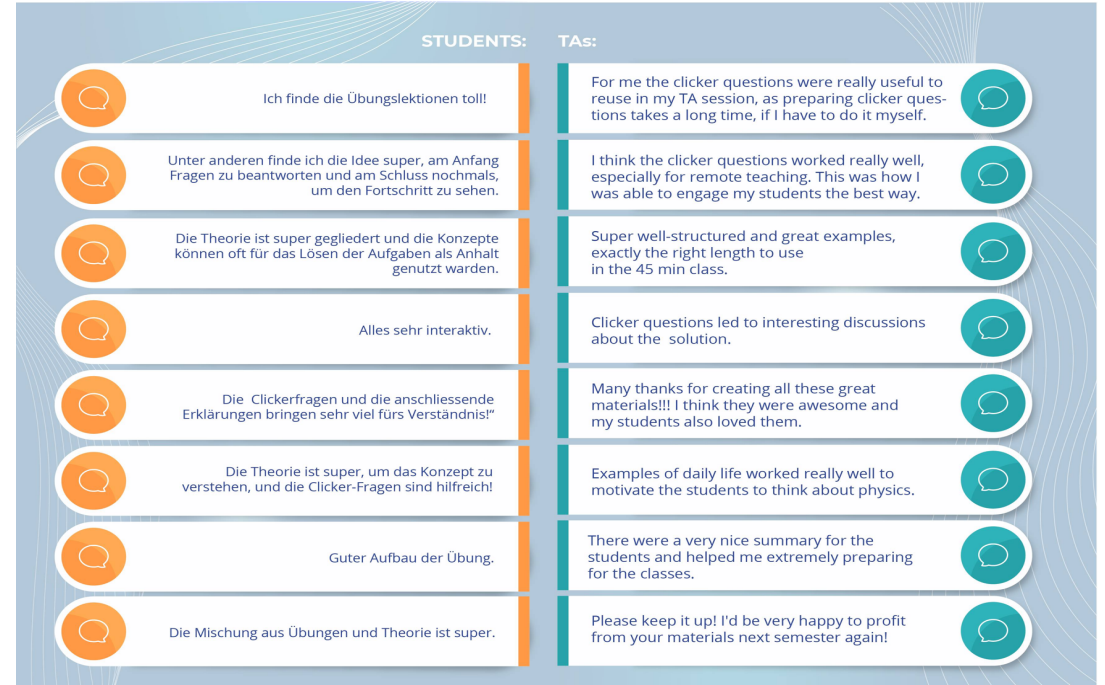

Figure 8. Further impressions from students and TAs.

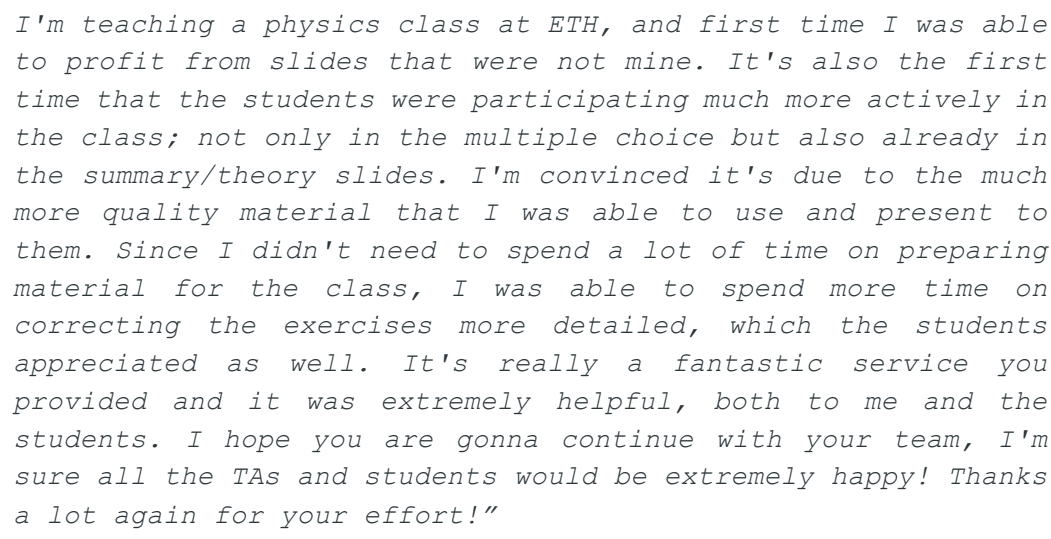

Figure 9. Comment by a TA.

\section{Summary and outlook}

We have developed a didactical toolbox of material for TAs who are teaching exercise classes for non-physics majors. The material of the toolbox was used by several TAs and we collected feedback from students and TAs from one focus group. Based upon the positive feedback received, we conclude that our material helped to engage the students and to 
enhance their learning. The toolbox played an important role in preparing high-quality exercise sessions, which both students and the TAs enjoyed together.

However, we realized that, besides providing teaching material, it is also paramount to establish a community of practice, where TAs can share and discuss their teaching experience. Fostering PCK (Pedagogical Content Knowledge) (Carlson et al., 2019) is as important as providing good teaching material.

This successful experience now has motivated us to continue the development of our material and to expand our focus to all of ETH Zurich exercise classes for introductory physics. In addition we will also focus on PCK and on social aspects in teaching.

\section{Acknowlegements}

The EPT project was supported by the ETH Zurich Rector's Impulse Fund.

\section{References}

Biggs J. \& Tang C. (2015). Constructive Alignment: An Outcomes-Based Approach to Teaching Anatomy. In: Chan L., Pawlina W. (Eds.) Teaching Anatomy. Springer, Cham, https://doi.org/10.1007/978-3-319-08930-0_4

Bondar V. et al. (2021). Engaging Physics Tutoring: a didactical toolbox for your exercise class, https://wp-prd.let.ethz.ch/WP0-CIPRF91493/ (retrieved Feb 2021).

Carlson J. et al. (2019) The Refined Consensus Model of Pedagogical Content Knowledge in Science Education. In: Hume A. et al. (Eds.) Repositioning Pedagogical Content Knowledge in Teachers' Knowledge for Teaching Science. Springer, Singapore. https://doi.org/10.1007/978-981-13-5898-2_2

Crouch C. H. \& Mazur E. (2001). Peer Instruction: Ten years of experience and results. American Journal of Physics 69, 970-977, https://doi.org/10.1119/1.1374249

Feldman G. et al. (2019). Collaborative Group Learning in a Swiss introductory physics class. Journal of Physics: Conf. Series 1286 012020, https://doi.org/10.1088/17426596/1286/1/012020

Gurlitt J. (2012). Advance Organizer. In: Seel, N. M. (Ed.) Encyclopedia of the Sciences of Learning. Springer, Boston, MA, https://doi.org/10.1007/978-1-4419-1428-6_157

Meredith D. \& Redish E. (2013). Reinventing physics for life-sciences majors. Physics Today, 66(7), 38-43, https://doi.org/10.1063/PT.3.2046

Oliveira P. C. \& Oliveira C. G. (2013). Using conceptual questions to promote motivation and learning in physics lectures. European Journal of Engineering Education, 38(4), 417 424, https://doi.org/10.1080/03043797.2013.780013

Rebello N. S. et al. (2007). Transfer of Learning in Problem Solving in the Context of Mathematics and Physics. In: Jonasson D. H. (Ed.) Learning to Solve Complex Scientific Problems, Routledge, New York, https://doi.org/10.4324/9781315091938-10 